\title{
How the Needs of Self-presentation are Related to the Social Media Fatigue of Chinese College Students on WeChat Moments
}

\author{
Xinhao Liu* \\ School of Journalism and Communication, Wuhan University, Wuhan, Hubei 430072, China \\ *Corresponding author. Email: 958613474@qq.com
}

\begin{abstract}
The needs of self-presentation have been confirmed by past studies as one of the factors leading to social media fatigue. Based on semi-structured interviews with twenty Chinese college students, this paper investigated how social activities on their WeChat Moments have changed, and explored how the demand for self-presentation has led to social media fatigue in this change. The trend of enlargement of the circle of contacts can be clearly seen on their WeChat Moments, through which the contacts who maintain "weak ties" with the users have gradually become the majority, causing the users to experience role conflict in social interaction. Therefore, the users have lost control of the "stage", and been forced to change their self-presentation strategies. Social media fatigue often occurs at this time. For more secure "audience isolation", they tend to migrate to other social platforms. Thus, this paper revealed a specific path of social media fatigue caused by the demand for self-presentation.
\end{abstract}

Keywords: social media fatigue, self-presentation, role conflict, WeChat Moments

\section{INTRODUCTION}

Social media fatigue has gradually become a hot topic in recent years. Past studies have tried to show that more and more social media users gradually fade out of social media participation because of social media fatigue [1]. As an academic term, social media fatigue usually refers to some subjective emotions reflected by users over time in the process of using social media. These include fatigue, worry, anger, disappointment and caution [2]. This reduces their demand or motivation for the use of social networks, even making some users tend to avoid social media participation [3]. Indeed, past studies have found that social media fatigue has resulted in "a myriad of" social media users to gradually stop engaging with social media participation [1].

Some scholars' researches on WeChat Moments in the past few years have shown that Chinese users are also suffering from social media fatigue on this social platform [4-6]. WeChat Moments, a social application operated based on the contacts of each WeChat account, is one of the most popular social platforms for acquaintances. According to the interim financial statements of Tencent Group in 2021, the number of global user active accounts of WeChat has exceeded 1.25 billion, a year-on-year increase of 3.8\% [7].

The research on social media fatigue is still in its early stage [1]. Existing papers are devoted to figuring out the causes of social media fatigue. Among the several possible causes, the motivation of self-presentation has been proved to be associated with social media fatigue $[4,8]$.

Self-presentation is a process. As E. Goffman observed: an individual acts in a completely planned way and expresses oneself in a predetermined way. The purpose of self-presentation is purely to put an impression on others and lead them to make the specific response one expects [9]. These kinds of behaviors can also be observed in online social activities. What is related to "self-presentation" is the concept of "performance", which Goffman defined as "a specific participant affects all activities of any other participant in any way on a specific occasion" [9]. In a social situation, there are many types of performances, including "idealized performance", "misrepresentation" and "mystical performance", which are the three means of self-presentation and impression management [9]. Wang argued that college students would also make use 
of the above three performance methods to show themselves in online social networking, and these performances may eventually increase their online social depression and contribute to social media fatigue [10].

Apart from some factors that may be related to the process, scholars have rarely chosen to explore the detailed process that how the motivation of self-presentation leads to social media fatigue. At least for now, it is hard to tell whether the social media fatigue is caused by the satisfaction of users' self-presentation needs, or is caused by the depression of this kind of needs. This paper explores this problem. This article investigated how the social activities of Chinese college students' WeChat Moments has evolved, and considered how those students' self-presentation leads to social media fatigue as well as the characteristics of this specific process.

\section{LITERATURE REVIEW}

The definition of social media fatigue is still controversial. Some studies attribute such "fatigue" to a kind of emotional performance. In their analysis of the characteristics of social media fatigue, Ravindran, Chua \& Goh concluded that social media fatigue can be defined as a subjective and multidimensional user experience, and in addition to fatigue, the user experience often encompasses worry, anger, disappointment, caution, as well as loss of interest or reduced demand for / motivation to engage in all aspects of social network use and interaction [2]. Similarly, after summarizing the views of many scholars, Dhir et al. defined social media fatigue as the feeling of exhaustion caused by an overload of various technologies and information exchange [1].

Other scholars posited other definitions, which include references to user behavior or potential behavior. For example, in a study on Facebook, scholars Bright and others suggested that social media fatigue results when users want to escape media participation because they feel swallowed up by the information they consume via social software [3].

However, some scholars do not agree: they do not include behavior-related factors in the definition. Scholar Zhao said that there is a huge inconsistency between users' emotional attitude towards social media and their behavior of using [11]. To be more specific, social media use is not just a personal matter, but is closely related to work, learning and image management "Young people may not give up using social media under any circumstances, and they are even very cautious about turning off some functions " [11]. A generally agreed conclusion is that social media users tend to choose to migrate to other social media platforms rather than simply give up online social networking [12]. Ravindran, for example, noted that not all social media fatigue experiences lead to a reduction in users' social network activities [2]. That latter observation has guiding significance for this research, because it is necessary to understand the range of how users deal with social media fatigue to meet their self-presentation needs.

For the same reason, this paper concludes that social media fatigue is a negative user experience, which can often be observed when the users of a certain social media application reach a certain degree of maturity [2]. This kind of experience includes worry, anger, disappointment, caution and so on. When users suffer from social media fatigue, their motivation to participate in online social networking decreases, but they may not have corresponding behavior.

As mentioned above, most of the existing articles on social media fatigue are devoted to exploring and proving the causes of fatigue. Those studies use questionnaire data and in-depth interviews based on grounded theory to come to their conclusions; Among them, SSO (stress-strain-outcome) is the most common theoretical framework in that questionnaire-based research. SSO "links stressors with outcomes and places strain as the mediating factor." [13]. The "stressors" and "strain" are the "causes" that scholars search for. One study by Zhang et al. explores the interaction between "stressors" and "strains" [14], distinguishing the categories and functions of the factors that can lead to social media fatigue, that is, their position in the logical chain. The direct factors, which are "strains" are a series of emotions and feelings, they write, such as low sense of achievement, emotional anxiety, decreased interest, social anxiety and emotional exhaustion. Many widely discussed factors that are possible to contribute to media fatigue are sometimes only sources of strains, but not the reasons that cause fatigue itself. These sources include FOMO (fear of missing out), forced use and so on, which will be introduced in the following paragraphs [14]. The importance of Zhang's article is that it reveals the rich interaction between various factors that may lead to social media fatigue. Some factors may rely on others at a particular time to act on social media fatigue. For example, FOMO causes users to experience emotional anxiety and tension, which further lead to social media fatigue.

Other scholars noted that the causes of social media fatigue are very diverse [3]. some pointed to the technical level of social media platforms, arguing that the anonymity and presenteeism of social media are both "stressors", and so may greatly affect social media fatigue [15]. They noted that social media applications with poor anonymity, such as WeChat (because people are asked to use it at work), let work invade daily life, resulting in social media fatigue [15]. Presenteeism, which refers to the extent to which social media enables 
users to access information, leads to privacy intrusion. Some instant messaging social media applications tell the senders whether the message recipients have read the message they send, which makes some users feel that their privacy and freedom of use are not protected, and indirectly increases the degree of social media fatigue [15]. from a technical perspective, Xiao and others argued that both the connectivity and the immediacy of feedback of social media applications are the key factors leading to fatigue. However, these two features do not play a direct role. They are related to social media fatigue through some other factors which would be discussed in the following paragraphs.

Some of the most widely discussed factors that can contribute to social media fatigue include information overload, forced use, privacy concerns and FOMO, as well as the self-presentation that would be focused on by this article. Among them, the pressure of information overload greatly weakens the enthusiasm of users to use social media, resulting in the formation of online social media fatigue intention [16]. Whelan and others believe that some users use social media precisely because of their "boring tendency", but they fall into the trap of information and communication overload, and finally suffer from social media fatigue [13].

Besides, compulsive social media use is described as an important factor for social media fatigue of adolescent social media users [1]. However, this conclusion is controversial. An article concentrating on the use of WeChat during the epidemic concluded that there is no obvious positive correlation between social media forced use and social media burnout, and the relationship between them is not completely clear. Similarly, whether FOMO has a sufficiently important impact remains to be identified.

Privacy concerns are considered to have a positive impact on social media fatigue [17]. It is worth noting that privacy concerns are actually related to self-presentation. According to a study, privacy concerns are actually caused by a series of factors, including "cognitive-emotional concerns about the personal image" [17], as self-presentation is described as a series of actions that try to influence your image in others.

Significantly, from the current literature, there is a logical correlation between users' motivation of self-presentation or impression management and social media fatigue. That is, the motivation of self-presentation is a factor leading to social media fatigue $[4,8]$. A study indicated that, on the WeChat Moment which relies on the real social network, users have a high degree of interpersonal connection and interest correlation, which makes users have to pay attention to the content of self-expression and the evaluation of self-image by others. When self-expression may bring negative evaluation, users will give up self-expression to maintain a positive self-image. The importance of this conclusion is that it revealed one of the possible paths of social media fatigue caused by the motivation of self-presentation [6].

In addition, an article focusing on the special group of media practitioners finds out that the motivation of impression management led media practitioners to create the image of "hard-working, friendly, positive, personalized and loving the company" [18] on social media, thus having to increase the proportion of using social media in non-working hours [18]. The value of the above article is that it revealed that the motivation of self-presentation may lead to social media fatigue by forcing users to maintain social media use for a long time (social media forced use). It suggested the idea that the motivation of self-presentation also interacts with other factors related to social media fatigue.

At present, there are at least two factors proved to be related to the motivation of self-presentation and social media fatigue, the enlargement of social media circle of contacts and the role conflict. For instance, scholars Hong \& Duan studied the impact of the enlargement of WeChat's circle of contacts on the online social self. They presented that the WeChat Moments was originally designed as circle social environment in which only users who are each other's contacts can interact. When more and more friends in the environment come from different small circle layers, users' privacy boundaries would become blurred, thus harming their self-awareness [4]. Specifically, friends from different backgrounds and living in different environments may have completely different interpretations of a post published by a user. Some interpretations inevitably deviate from the original meaning of the post, and even cause the publisher of the post to receive uncomfortable comments. Therefore, users may have to reduce their expression on WeChat Moments due to the need of self-protection [4].

At the same time, the enlargement of users' circle of friends on WeChat has led many "friends" who are not familiar with the account owner to obtain the right to "be present". To some extent, the comments made by users on WeChat Moments have become public secrets, and their contents may be forwarded to more people within a second. This also brings more difficulties to the self-presentation of users on WeChat Moments [5]. It is worth noting that in the articles of Hong \& Duan and Jiang, the social dramatic theory proposed by E. Goffman has also been used to explain the relationship between the enlargement of users' circle of friends and social media fatigue. For instance, scholars Hong \& Duan analyzed that users' instinct of impression management forces them to treat their WeChat Moments which are full of uncertainty as "front region" instead of "back-stage", thus increasing the extent of their sense of tension [4].

However, from the current research, what type of 
friend-relationship is the key harm for users to lose confidence in self-presentation on WeChat Moments remains to be further studied.

Another cause of social media fatigue related to self-presentation is the role conflict experienced by users on social media, for the reason that role play is a crucial part of self-presentation. Previous studies have proved that role conflict has a significant impact on social media fatigue and dissatisfaction, and the greater the pressure of role conflict perceived by users, the stronger the feeling of social media fatigue and dissatisfaction [19]. As an example, scholars Bossio and Holton conducted an interview based on grounded theory with 39 journalists from the United States and Australia [20]. they have found that this group was facing social media fatigue caused by huge role conflict. The reason is that it is difficult for them to balance personal and professional identity on the social platform, which makes it difficult to express themselves through publishing personalized content, while media organizations require them not to leave the social network at will [20].

Besides, user personality, which sometimes has an impact on social media fatigue, cannot be ignored, for the reason that users' personality affects the extent and characteristics of their self-presentation. Generally speaking, neurotic personality is positively correlated with social media fatigue [21]. one possible reason is that users with such personalities are more likely to feel privacy violations on social media platforms [15]. And the negative correlation between extroverted personality and social media fatigue is the most obvious [21].

For the theme discussed by this article, that is, the relationship between the motivation of self-presentation and social media fatigue, previous studies have summarized three related factors: enlargement of circle of contacts on social media, role conflict and users' personality. However, the specific path of the motivation of self-presentation leads to social fatigue; how do tired users tend to solve their emotional problems; and what kind of interpersonal relationship on social media plays the key role contributing to the frustration of users' self-presentation remain to be further explored. In short, in previous studies on social media fatigue, the motivation of self-presentation has not been regarded as a key point for analysis, which left room for further research.

\section{RESEARCH QUESTIONS}

Previous studies have proven a general causal relationship between self-presentation and social media fatigue. However, when it comes to specific groups, the relationship must be tested again and directionally. Therefore, the first research question is:

1. In Chinese college students. Does the need for self-presentation really lead to social media fatigue on WeChat Moments to some extent? (the reasons for choosing Chinese college students and WeChat Moments as the research objects will be explained later).

The gap left by previous studies is that the exact logical chain of how the motivation of self-presentation leads to social media on a specific social platform is unclear yet. Hence, the second and third research question are:

2. In what way does the motivation of self-presentation related to social media fatigue in the social interaction of Chinese college students on WeChat Moments? And what are the factors that play a role in the formation of this relationship?

3. How do these college students who suffer from social media fatigue seek for a solution? What social needs do the several solutions reflect?

\section{RESEARCH DESIGN}

\subsection{Methodology}

This article adopted qualitative semi-structured interviews to carry out the research. Most of the published articles focusing on social media fatigue prefer quantitative questionnaires or qualitative interviews based on grounded theory. The reason why this article did not choose to adopt the questionnaire is that the research topic no longer focuses on discovering or confirming the various reasons for social media fatigue. The research attempts to reveal the detailed logical relationship between the motivation of self-presentation and social media fatigue, so that qualitative interviews are more appropriate.

This topic has rarely been explored in depth before, so it is necessary to have an in-depth understanding of the psychological activities of the respondents, which is exactly the advantage of an interview methodology. To some extent, the interview can be regarded as the product of cooperation between researchers and respondents. Interviewees actively participate in the interactive process, rather than only passively responding to researchers, although it is still a dialogue "being guided (by researchers)" [22]. This gives respondents more opportunities to explain their stories so that researchers can understand the rich inner world of respondents [22]. For the unclear logical chain between the motivation of self-presentation and social media fatigue, this detailed explanation from respondents behaves better than the blind hypothesis made by researchers. Besides, the interview is also possible to produce some unexpected information "falling from the crack" of formal information [23], which may give birth to enlightening concepts. 
there are many disadvantages of interviews though. The interviewers may record what they want to see rather than the facts [23]. Researchers may inadvertently guide respondents to discuss their research themes, and in so doing ignore the perspectives and views of respondents [22]. A way to avoid this is for researchers to ensure the high participation of respondents in the interview conversation.

Interviews can be divided into three types: structured, unstructured and semi-structured. Structured interviews make use of a form of question list [23]. During the interview, the researcher lists all the questions to be answered in a questionnaire prepared in advance. Then, the questions are put forward in order, and the answers are aligned with that predetermined sequence and classification [22]. Unstructured interviews allow respondents to express their overall views on the survey subject without detailed questions. Unstructured interviews also allow interviewers to expand the scope of inquiries according to the new ideas raised by the respondents; unstructured interviews tend to produce a great deal of explanatory information [23]. Semi-structured interviews require researchers to prepare an interview outline and allow respondents to raise important questions themselves. Unstructured and semi-structured interviews are often called in-depth interviews [22] — but the boundary between the three types is often not so fixed and clear [22].

The above comparison identifies the reason why this paper did not choose structured interviews. The defect of structured interviews is that they can only obtain superficial data, which is not flexible enough and suppresses the enthusiasm of respondents [22]. At present, as mentioned above, people's research on social media fatigue is still in its infancy. Moreover, at this stage, few relevant studies reflect the specific logical relationship between the motivation of self-presentation and social media fatigue. Therefore, the research on this topic has to rely more on the respondents to provide enlightening information. From this perspective, structured interviews are inappropriate.

In addition, unstructured interviews and other qualitative research methods, including grounded theory, are not suitable for the themes and problems identified in this study. Although not fully discussed, previous research has teased out factors that can link the motivation of self-presentation to social media fatigue, such as the enlargement of the circle of contacts and role conflict. As mentioned above, the social dramatic theory has also been widely used to explain the relationship between this series of phenomena and social media fatigue. In other words, it is reasonable to adopt the social dramatic theory to the interviews and data analysis. However, grounded theory is a bottom-up induction and research process, which extracts theory from empirical data rather than guiding research with a certain theoretical framework or premise [14]. To some extent, for this research topic, it is necessary to make full use of some theoretical conclusions obtained from previous research. Therefore, on the whole, the semi-structured interview is the most suitable method. The enlargement of WeChat's circle of friends and role conflict have been confirmed, and the factors related to the research topic will be tested in the interview.

\subsection{Sampling \& Data collection}

This paper chose WeChat Moments as the "field" of investigation. One possible reason has been mentioned above, that WeChat is one of the most popular communication applications for acquaintances in mainland China. Meanwhile, WeChat Moments is a social platform established based on each user and all his or her contacts, and it is built into WeChat app. Its utilization rate has had a certain downward trend in recent years, which is considered to be related to an increasingly extensive social media fatigue [4].

This paper selected Chinese college students as the research object. Before interviews, it is needed to include a process of collecting samples [23], and the sampling process refers to the determination of respondents [22]. According to different research purposes, the sample group selected by researchers can be "most extreme individuals", "the most critical individuals", "people with large differences from each other", a group of "homogeneous people" and so on [22]. As of December 2020, only 9.3\% of Chinese mainland netizens have a college or graduate education. Meanwhile, only $13.5 \%$ and $17.8 \%$ of Chinese Internet users are from groups aged 10-19 and 20-29 respectively [24]. Judging from the average educational level and age structure of Chinese netizens, though, college students are indeed not a broadly representative group. It's more of a highly characteristic and homogenous group.

However, choosing this group as the research object may be more conducive to exploring the relationship between self-presentation and social media fatigue for two main reasons. Firstly, the age of undergraduate students in Chinese universities is generally between 18 and 23. In China, "digital natives" are generally defined as the group born after China's access to the Internet in 1994 [25]. Most of these people were born around the millennium, who are typical GEN-Z and digital natives. They grow up in the environment surrounded by ubiquitous information technology [26], so digital life is of great significance to them. To some extent, the WeChat Moments accounts have become the "Avatar" of college students in cyberspace, by which they present themselves and manage their own image in various ways [10]. Secondly, before and after entering the University, the number, and types of contacts in a student's WeChat Moments would inevitably show 
obvious differences. According to the social dramatic theory, WeChat Moments can be regarded as a "stage" for users to "perform". When the "stage" changes, users' motivation of self-presentation and their "performance" strategy will also change to some extent. Then, by comparing the users' self-presentation in the two periods, the possible correlation between social environment, motivation of self-presentation and social media can be found.

Since social media fatigue involves individual subjective emotions, it must be known and confirmed in advance that the interviewee is indeed experiencing or has experienced the feeling of social media fatigue. Therefore, sampling is difficult to be probabilistic. Besides, the sampling selection of interviews can be probabilistic or non-probabilistic. Since social media fatigue involves individual subjective emotions, it must be known and confirmed in advance that the interviewee is indeed experiencing or has experienced the feeling of social media fatigue. Therefore, sampling is difficult to be probabilistic.

Snowball sampling is included in the several methods of non-probabilistic sampling. An example of this method is that, after interviewing an interviewee, the researcher puts forward "can you introduce me to your peers?" [22]. By snowball, highly homogeneous interviewees can be obtained in a short period. It can also avoid a case that the interviewees are limited to those who already know the researcher, which will negatively affect the reliability and validity of the article. Therefore, this paper started with 4 interviewees known to the researcher and used the snowball method to obtain more interviewees.

The repeated answers to the question "how many interviewees are needed" is "depending on the situation" [27]. The conclusion of this paper was based on 20 interviews, 16 of which were obtained by snowballing.

\subsection{Data analysis}

During and after the interview, the qualitative data obtained was encoded twice. Coding and classification are key links in the process of qualitative data analysis. Coding is the process of extracting themes or concepts from the original data, often represented by symbols or numbers, while classification is the process of classifying research data into various codes [22]. The advantage of coding and classification is to simplify the data, so that researchers can quickly find the information they need, and compare the same coding [22].

The two layers of coding adopted in the paper were primary-cycle coding and secondary-cycle coding. Primary-cycle coding, similar to open coding mentioned in grounded theory, encodes the collected data intensively and carefully until the coding reaches saturation. In the article, the number and type of contacts on WeChat Moments of the interviewees, the emotions they experience in using the WeChat Moments, and the categories of content they publish daily were the topics of primary-cycle coding. The secondary-cycle coding is roughly similar to the so-called axial coding proposed by the grounded theory. Secondary-cycle coding establishes the relationship (causality, time sequence, parallel relationship, logical correlation, etc.) between the codes formed by primary-cycle coding, combining the related codes to establish a large category, and uses a more abstract concept to name this category [22].

\section{INTERVIEWING \& CODING}

As table1 shows, twenty interviews were conducted according to the established procedures. Telephone recordings were created, ranging from 37 minutes long to 102 minutes long. The 20 pieces of data were divided into two encoding processes.

Table 1 basic information of samples

\begin{tabular}{|c|c|c|c|c|c|c|c|c|c|c|}
\hline & sample A & $\begin{array}{l}\text { sample } \\
\text { B }\end{array}$ & $\begin{array}{l}\text { Sample } \\
\text { C }\end{array}$ & $\begin{array}{l}\text { sample } \\
\text { D }\end{array}$ & sample E & Sample F & sample G & sample H & sample I & sample J \\
\hline Gender & Female & Female & Male & Female & Female & Male & Male & Female & Male & Female \\
\hline Age & 18 & 19 & 19 & 19 & 21 & 20 & 18 & 19 & 20 & 19 \\
\hline Region & Wuhan & Fuzhou & Xiamen & Wuhan & Taiyuan & Beijing & $\begin{array}{l}\text { Shenzhe } \\
\mathrm{n}\end{array}$ & Wuhan & $\begin{array}{l}\text { Chengd } \\
\mathrm{u}\end{array}$ & $\begin{array}{l}\text { Changsh } \\
\text { a }\end{array}$ \\
\hline \multirow[t]{2}{*}{$\begin{array}{l}\text { duratio } \\
n\end{array}$} & 46 & 37 & 51 & 84 & 35 & 39 & 41 & 53 & 57 & 66 \\
\hline & Sample K & $\begin{array}{l}\text { Sample } \\
\mathrm{L}\end{array}$ & $\begin{array}{l}\text { Sample } \\
\text { M }\end{array}$ & $\begin{array}{l}\text { Sample } \\
\mathrm{N}\end{array}$ & Sample O & Sample P & Sample Q & Sample R & $\begin{array}{l}\text { Sample } \\
\text { S }\end{array}$ & $\begin{array}{l}\text { Sample } \\
\text { T }\end{array}$ \\
\hline gender & Female & Female & Female & Male & Female & Female & Male & Male & Male & Female \\
\hline Age & 19 & 19 & 20 & 18 & 19 & 19 & 21 & 23 & 20 & 19 \\
\hline Region & Shanghai & Haikou & Qingdao & Beijing & Beijing & Wuhan & Guangzh & Xi'an & Tianjin & Nancha \\
\hline
\end{tabular}




\begin{tabular}{lllllllllllll} 
& & \multicolumn{1}{c}{} & ou & & $\mathrm{ng}$ \\
\hline Duratio & 69 & 76 & 34 & 36 & 48 & 65 & 62 & 102 & 68 & 28
\end{tabular}

Before analyzing all the primary-cycle and secondary-cycle codes, the characteristics and structure of social networking based on WeChat Moments need to be further explained. As WeChat itself is an instant messaging application, WeChat Moments is largely considered to be a network field based on displaying social relations where "strong ties" and "weak ties" coexist [28]. In 1973, Mark Granovetter first put forward two relative conceptual categories of "strong ties" and "weak ties" in the way of theoretical definition. Generally, strong ties are regarded as stable and profound social relationships, while weak ties are more flexible and extensive social relationships compared with the former [29]. WeChat Moments is defined as an "acquaintance social platform" — in other words, although the social networking based on this platform is public, people who participate in the social activities of this platform are known to the account owner. The platform connects individuals via both "strong ties" and "weak ties".

This implies that it is difficult for users to show eternal consistency in self-presentation on WeChat Moments. For the reason that previous study discusses that people's degree of impression management is low on "strong ties" relationship-based social platforms. On the other hand, users are not familiar enough with their "weak ties" contacts on the WeChat Moments, so they need to carry out impression management. Therefore, on social platforms dominated by "weak ties", the degree of impression management increases [30].

This study identified six categories of secondary-cycle codes, which are frequency, personality, content, factors, contacts and solutions. For instance, the "frequency" of secondary-cycle code is classified as the frequency of users' publishing content on the WeChat Moments before entering the University and in the recent period. These different frequencies are recorded as different primary-cycle codes, such as "multiple times a day", "once a day", "multiple times a week", "once a week" and "no obvious rules". Eighteen interviewees have said that within two or three years after attending a university, their frequency of publishing on WeChat Moments decreased significantly.

There were three categories of content that interviewees considered most hesitant to post. These are opinionated content (book reviews or comments on events), emotional catharsis (especially negative emotions) and daily sharing. This constant hesitation has brought a feeling of fatigue. Subsequent in-depth analysis will explain the close relationship between these types of content and the need for self-presentation.

By comparing the number and types of contacts, the article found that the circle of "friends" (contacts) of these users has been obviously enlarged. Firstly, in terms of number, the average number of contacts on WeChat accounts of the 20 interviewees has exceeded 450. The number of contacts of five of those exceeded 500, far more than Dunbar's number (150). By comparison, this number rarely reached 100 before they entered the college. Secondly, the types of WeChat contacts have also been widely expanded. The contacts often include teachers, classmates/schoolmates, dormitory managers and fruit stall owners, etc. In the primary-cycle codes, all the contacts were reclassified into three categories: "strong ties", "weak ties" and "lack of relationship". In college life, the growth of contacts with account owners with weak ties is the most significant.

Before presenting the result of coding, it must be clear that individual self-presentation, whether in real life or the network society, is a kind of "performance". "Performance" refers to all the behaviors that an individual show when continuously facing a group of specific observers and has some influence upon those observers [9]. Then, in the process of "performance", there is a "front region" where individuals perform. Front region refers to the part of a performance that regularly defines the situation for the observers in a general and fixed way [9], containing two aspects: "setting" and "personal front region". The former provides scenery and props for people to perform everywhere in the stage space. Generally speaking, the setting is often fixed, so people have to be in the appropriate setting to start their performance, or they would feel unaccustomed [9]. And the latter includes a person's appearance and manner [9].

The category "Factors" under the secondary-cycle code are the most noteworthy. "Factors" records three main self-presentation strategies carried out by the interviewees that may lead to social media fatigue. Consistent with previous studies, the self-presentation of Chinese college students on the WeChat Moments includes "idealized performance", "misrepresentation" and "mystical performance" [10]. They are forms of self-presentation, though there are more than three available. For example, an interviewee claimed that "I sometimes want to look cold". As a consequence, the interviewee took active impression management measures and deliberately published unpredictable content. Apparently, it is an idealized performance. However, the idealized performance also limits the desire for expression. If people want their performance to meet the ideal standards, they must abandon or hide behaviors that are inconsistent with these standards [9]. Sometimes people don't realize they are performing. 
Some interviewees recalled feeling that the content they wanted to post were so childish that they ended up not posting them. In fact, they didn't realize that they were simply afraid of being considered childish by others. People keep planning in the performance, but they do not realize it accordingly [9].

Some sensitive users have realized that they did have a lot of worry before publishing some content. Therefore, their impression management becomes passive due to their insecurity about the online social environment. They dare not to cause the dissatisfaction of the audience.

Besides, misrepresentation is used to maintain relationships that users see as fragile. The audience's certain acceptance tendency put the performers in a misunderstood situation, making the performers cautious in all the expressions in front of the audience. Then, this tendency for acceptance also puts the audience in the position of being deceived [9]. Misrepresentation is used to deal with the relationship between some users and relatives who are relatively estranged from the former, so as avoid leading to a bad view of the users because of some posts.

\section{FURTHER ANALYSIS}

\subsection{Enlargement of circle of contacts and role conflict}

by comparing the changes of the interviewees' self-presentation strategies before and after entering the University and their attitudes towards the social networking of WeChat Moments during the two period, the article found that the enlargement of the circle of contacts have made their WeChat Moments no longer the "back-stage" where they can get rid of complex interpersonal relationships and relax themselves, but the "front region" where social environment is hard to predict.

For Chinese students (including high school students or college students), places like classrooms, student dormitories and canteens are the main social occasions in their daily life. In such a "stage", the interviewees not only need to socialize, but also need to face all kinds of emergencies or accidents. This means that "scripts" and "props" are often not controlled in the hands of performers. In real life, people's self-presentation is difficult to achieve the ideal state. Scripts and props are part of the stage "setting" [9]. Goffman believes that no matter who wants to take a specific stage "setting" as a part of the performance, he or she must be in the "appropriate" setting to start their performance [9]. The "personal front region" is also difficult to be artificially changed, because it mainly includes a person's "appearance" and "manner", which are difficult to disguise or hide [9]. For most research objects, WeChat
Moments used to be an idealized "stage". The constraints brought by the originally fixed "setting" and "personal front region" have been relieved. For example, individuals get rid of the constraints of the body, appearance and so on, and any image is presented in the form of symbols. At the same time, users only need to focus on social activities on the WeChat Moments. This is not a real-world integrating various complex affairs. Here, "setting" can be partially controlled by the user (for example, setting avatars, nicknames, and "tickles"). This "stage" provides performers with more ideal conditions for impression management.

For other interviewees, the ideal form of WeChat Moments is their "back-stage" for self-presentation. The back-stage is considered to be a place where performers consciously or unconsciously create the opposite effect of the "front region" [9]. At the same time, the most critical secrets of the performance can be seen on back-stage, where people can get rid of the trouble around them and relax [9]. they regard real life as a "front region" and expect that WeChat Moments can be a back-stage.

However, after these students entered a new environment, the circle of their online contacts enlarged. This is not only reflected in the change of the number of contacts and the diversification of contact types, but also a change of structure. In the new social structure, "weak ties" have become the main type of relationship on their WeChat Moments.

Before entering the college, the social scope of the account owner was limited, the contacts on the WeChat Moments maintained a relatively stable relationship with users, and the mutual relationship between these contacts was limited except for the part known by the account owner. For instance, $\mathrm{B}$ and $\mathrm{C}$ are classmates of the account owner, and $\mathrm{D}$ is the neighbor of the account owner. The relationship between $\mathrm{B}$ and $\mathrm{C}$ is certain and well known to the account owner. The relationship between D and B or C can be ignored, for the reason that they do not know each other. Hence, they would not interact in the social space of the account owner. In other words, the account owner can fully grasp the situation of the "audience" of the stage.

After entering the University, with the continuous socialization of personal life, the social circle of individuals expands rapidly, so that the people who can participate in the social activities on the account owner's WeChat Moments expand from a relatively single group of acquaintances to a large number of contacts with different types of relationship with the account owner. These groups include not only acquaintances that users have known in the past, but also contacts added due tostudying, work or activities. There are also some contacts, including classmates, colleagues or social figures who have once had an online interaction with the interviewee but have not actually met them in the real 
world. Interviewees added most of them as WeChat contacts from the needs of work (including student union, student associations, etc.). More importantly, there is a complex relationship network among users' contacts, which is far beyond their knowledge or grasp. For instance, $\mathrm{b}$ is a roommate of the account owner, $\mathrm{c}$ is the colleague of the account owner in the student association, and $\mathrm{d}$ is an unfamiliar classmate from the same college as the account owner, who is also one of the leaders of the student union of the college; e is a teacher of the account owner. B may be very close to d, but the account owner is not quite sure about it; c may also have been a student of e. In other words, the relationship between them is complex and hidden. Although performers are aware of it, they often find it difficult to grasp it.

Under the complex relationship, the users performing on the stage named "WeChat Moments" could not grasp the general situation of the audience off the stage, which led them to fall into role conflict. This kind of role conflict is embodied in that, for instance, they do not know whether they should play as the audience's close friends on the stage or just play ordinary classmates who are not familiar with the audience. There is a certain matching relationship between their hesitation in self-presentation strategy and their psychological entanglement. This hesitation is embodied in the trade-off between self-exposure and privacy protection sometimes presents the form of "misrepresentation" or "mystical performance" in behavior. It should be emphasized that self-exposure cannot be equated with self-presentation, it is only one form of self-presentation. The latter mainly refers to a situation where a person guides the observer to form a certain impression of him or her [9]. Unable to identify their relationship with the audience, these interviewees who hesitated between self-exposure and privacy protection could not predict the effect of self-presentation, and finally lost the balance of self-presentation.

By analyzing the coding, this article found that social media fatigue is most likely to occur when self-presentation has been out of balance. The word "habit" appears frequently in the interview text. The interviewees had developed certain social habits before entering the University or in the process of starting college life. This habit has had a great impact on their social behavior. It imperceptibly promotes users to complete a social behavior they are used to. This is similar to what E. Goffman called "routine procedure": performers perform on a stage fixedly and regularly [9]. The new social environment makes performers lose control of the stage. They have to form new expression habits, which are reflected in the frequency and type of content. As the encoded data shows, their frequency of posting on WeChat Moments has decreased significantly. Meanwhile, the content of emotional catharsis type has become less.

Users consciously or unconsciously form new self-presentation strategies, and social fatigue often appears at this time. Because of the replacement of old and new habits, users face very contradictory moods when publishing on WeChat Moments. For instance, some users used to release some remarks expressing negative emotions in order to vent their unhappiness in real life or express their dissatisfaction with a specific person. Since they can no longer predict the audience's response to such content, they form a mood of "want to post but dare not to", which leads to a certain degree of disappointment, anger, caution, anxiety, and depression. And these emotions are typical of social media fatigue [2].

\subsection{Peers in "Weak ties"}

This article found that the peers who share a "weak ties" relationship with users on their WeChat Moments are the direct group leading to their social media fatigue. To some extent, the communication on WeChat Moments has the purpose of obtaining an emotional reward, this is, uses want feedback on their published content [5]. At the same time, due to the strong Intertextuality between the content published by the users and their daily living environment and events, they hope that a group of contacts with certain relevance to the environment and events involved in their published content can respond accordingly. In addition to some familiar friends, peer groups mainly connected with account owners by "weak ties", such as the students from the same school who are not familiar with each other, or colleagues from other departments who are organized with users on campus, have just built the most complex relationship network after the enlargement of circle of contacts, which makes it difficult for the account owners to accurately grasp the situation of the stage. Therefore, it falls into the difficulty of impression management.

Users cannot perform self-exposed and mystical performances in the same social field at the same time. Some interviewees believe that these unfamiliar peers who lived in the same field have potential interest associations or conflicts with themselves. When they cannot grasp the attitude of these unfamiliar peers towards them, performing self-exposed may bring trouble to them. However, the mystical performance would limit their desire for expression.

In real life, people can adopt "audience isolation" to deal with the problem of being unable to grasp the overall situation of the audience. In other words, when people perform specifically, they can avoid the audience they do not want to see. Through this isolation, the performer can ensure that the audience watching his specific role-play at this time will not be the audience 
when he performs another role under another stage setting [31]. If people fail to isolate the audience and the outsider inadvertently sees a performance that is not aimed at him or her, there will be problems in impression management [9]. This is exactly the dilemma the interviewees encountered on WeChat Moments: there is only one stage, making it difficult for the audience to be effectively distinguished. Isolating the audience is a measure to control the "front region". If this control cannot be maintained, the performer will not know what role he should play at one time and what role he should play at another time [9]. In other words, they fall into role conflict, which makes the performance difficult to succeed.

Some other contacts are not of a similar age and are linked to users mainly by "weak ties", which makes interviewers fall into the difficulty of impression management, thus affecting their user experience towards WeChat Moments. These contacts include the distant elders in the users' families. The users should have played a mystical performance or misrepresentation for them, but this also limits them to express to their close friends. In order to avoid role conflicts, they sometimes have to publish through the "selected groups" function of WeChat, which can prevent some contacts from browsing specific content published by users. But in this way, the complexity of performing on the social stage and the fatigue of users have been greatly improved [31].

The conclusion above is not consistent with some previous studies. For instance, scholar Jiang believes that the groups on WeChat Moments that cause social media fatigue are mainly strangers added by users occasionally, including salesman and small businesses [5]. However, this paper believes that from the perspective of the needs of self-presentation, the trouble caused by this type of contact with users is not obvious. This may be because the specific problems discussed in the previous research and this paper are different. These contacts who send small advertisements will indeed make users feel bored, because they overload the users' feel bored, because they overload the users' WeChat Moments with the meaningless information. However, interviewees said that their active self-presentation on the WeChat Moments was not affected too much by such contacts, for the reason that these strangers who are unlikely to develop further relationships with them in the future inhibit their attention to privacy. Individuals tend to disclose more information while diluting privacy concerns [32].

To some extent, strangers are at least audiences that make performers feel safe, so that performers do not have to really grasp their situation. In other words, due to the low correlation between strangers and performers' daily life (now and in the future), users are free to present themselves in the way they desire, and do not have to worry about "the terrible accident of being caught at the crime scene" [9]. it is preliminarily judged that the process of users expressing themselves to strangers is actually a compromise of partially or completely abandoning contextual relevance and obtaining a sense of freedom and security of self-presentation.

\subsection{Solutions}

Interviewees who have lost their balance of self-presentation and social media fatigue have generally adopted three solutions. The first is to migrate to other platforms, the second is to set "selected groups", and the third is to give up taking measures.

Migrate to other social media apps: the stage is not easy to create, but easy to choose [9]. "Migrate to Weibo" has become the choice of most interviewees, and it is generally considered to be the best method. Weibo is a highly opened social platform of the Chinese Internet, and it is also the most extensive social channel for strangers in China. these strangers share no future intersection with the account owner, although they may be able to see the content published by the account owner. Interviewees felt they could perform more calmly on Weibo than WeChat Moments. They have known that what they express can be seen by many people, but at the same time, they do not have to worry about role conflict and unpredictable expression effects. This may be because Weibo is not an instant messaging social application that has to be used daily, therefore users can better grasp and even select the audience. The interviewees often only let their close friends know their Weibo account, so their "fans" only contain these friends who are allowed to watch their performances and some strangers who have nothing to do with them. At this level, the account owners have actually completed that "audience isolation" and stages have returned to a controllable state.

Migrating to other platforms is a process for users to reselect "front region" and "back-stage" in the network society. As mentioned above, some interviewees tried to regard WeChat Moments like the "back-stage" of real life, but they soon failed and fell into social media fatigue. Now some of them are trying to take the WeChat Moments is the front desk, where they often only carry out "idealized performance" and "mystical performance" and take other social applications, including Weibo, as the "back-stage", so as to regain the sense of control over self-presentation.

Setting "selected groups": this is the most commonly used solution which $90 \%$ of interviewees have tried. However, the effect is not as good as moving to other platforms. When they succeeded in "isolating" a part of the "audience" for which he was unwilling to perform, the performance returned to the "general and fixed" way 
again [9]. However, as analyzed above, "selected groups" add unnecessary preparation for the performance. For interviewees who have suffered from social media fatigue, "selected groups" are a kind of suggestion. It reminds the users that "stage" and "audience" are highly uncontrollable, and they are powerless. This hint once again affected their experience of social media.

Giving up taking measures: some interviewees are used to the imbalance of self-presentation on WeChat Moments. They hold back some specific content they originally wanted to release, such as posts to vent negative emotions, so they quickly entered a "mystical performance". This is also part of the uses' self-presentation strategies. Nevertheless, the users are forced to choose, and their needs of self-presentation are restrained. In other words, social media fatigue has naturally formed a kind of self-presentation. The result of this approach is often that there would be no "performers" on the stage of self-presentation".

\subsection{Personality}

Consistent with previous studies, the personality characteristics of these interviewees show a trend of homogenization. Most of them are delicate and good at thinking, and are very sensitive to some irritant factors in interpersonal relationships. This is similar to the "neuroticism" mentioned in previous studies $[21,15]$. In this paper, such personality traits make the interviewees fall into some. In this article, such personality traits have made the interviewees fall into some trouble of paying too much attention to the audience's reflection, which makes the performance itself easy to make people tired.

In the past, the classification of "performers" can be summarized as "sincere" and "cynical". The former may completely enter their role, and even sincerely believe that the image they present is the real one. The latter is generally considered not to be fully engaged in their role [9]. This paper found that almost all the interviewees are "sincere". A reasonable explanation is that they are more willing to present themselves sincerely and diligently. Therefore, they have more concerns and are more sensitive to the stage. In this case, they are more likely to fall into the fatigue of the performance. As a consequence, they pursue social freedom more strongly and tend to present themselves in a relatively real and reliable social environment. When they cannot judge the proportion of true expression and false expression in the social environment, they are more likely to get bored constantly.

\section{CONCLUSION}

Taking the WeChat Moments as the field of investigation, this paper found that, in the structural change of their circle of contacts, among Chinese college students there is a specific group suffering social media fatigue because the self-presentation strategies are disrupted.

WeChat Moments is a social media application carried in WeChat. Social media applications can be roughly divided into online social networking and mobile instant messaging applications [1]. WeChat Moments belongs to the former, while WeChat itself belongs to the latter. As a result, any contact added by users in WeChat will become one of the participants in the social space of their WeChat Moments.

Due to the above mechanism, great changes have taken place in the interviewees' WeChat Moments before and after college, that is, the enlargement of the circle of contacts. After enlargement, the WeChat Moments has undergone structural changes. "Strong ties" and "weak ties" coexist, and "weak ties" account for a larger proportion. These peers who connect with account owners with "weak ties" have become the main reason for their social media fatigue. Because, according to social dramatic theory, if users are compared to performers on the stage, they have long been used to presenting themselves in a "fixed and regular" way [9]. Before college, "strong ties" was the type of relationship that dominated the users' circle of contacts. Here, they can adopt self-presentation strategies depending on their needs almost all the time to find a balance between self-exposed presentation and privacy protection. For example, when they are unhappy, they can publish some content to vent their negative emotions on WeChat, and often get the feedback they want, in the form of approval or comfort. In that period, for them, this is the "back stage" of daily life, where they can show their hidden self and release the pressure. However, in the new social networking environment, too many peers who are not familiar with themselves are added as contacts. These users gradually found that the stage of their performance is no longer easy to control and feel uneasy about the audience. On WeChat Moments, "audience isolation" is more difficult to achieve than in the real world. As a consequence, users fall into role conflict. They do not know how to view the relationship between themselves and all the audience, and lose their confidence in predicting the effect of expression. Therefore, the desire for expression is gradually limited. At the same time, the self-presentation strategy was also disrupted, which made them a little upset and began to produce a series of emotions such as boredom, depression and anxiety, which marked the emergence of social media fatigue.

Most of these "performers" are "sincere". They try to present themselves more honestly, so they have more concerns about the audience. Performers have to exclude all expression forms that may overturn the impression of the performance in the process of their performance, so as to avoid misinterpretation 
inadvertently revealed by the performer by the audience [9]. This makes them more inclined to adopt a relatively safe way to do "audience isolation". That is to move to other stages they are more confident to control, such as Sina Weibo. Users let some of their close friends know their Weibo accounts, so they can freely present themselves while trying to ensure that their sense of security is not threatened. Weibo is also one of the most common social platforms for strangers. It may be safer to expose themselves to strangers, because there would be no intersection or conflict of interests between them in the future. However, people's self-presentation implies a desire to obtain emotional feedback through a meaning exchange, so the selected audience needs to have a certain correlation with the "stage setting". Although many people choose to socialize with strangers as a solution, there is a logical conflict hidden here, that is, it is difficult for strangers to form a certain correlation with "stage setting". And they are unable to give effective emotional feedback to users. Social interaction with strangers does enable users to present themselves effectively. But in fact, the need for emotional feedback has been gradually stripped off, thus users cannot be truly satisfied in self-presentation.

The article has confirmed that when uses' online self-presentation strategies are hindered, they are likely to suffer from social media fatigue. The reasons for this obstacle include the enlargement of circle of contacts and role conflict, which is caused by the former. Despite some achievements, the article still has many limitations, firstly, some studies have proved the positive or negative correlation between some factors and social media fatigue through regression analysis and correlation verification, using numerical statistics to calculate the strength of the correlation between these factors. However, this paper cannot verify the specific strength of the correlation among enlargement of circle of contacts, role conflict, self-presentation and social media fatigue. This is mainly due to the qualitative research method adopted in this paper. Although the specific process of the interaction of four factors is discussed, the process cannot be digitized. From the perspective of interviewees themselves, they believed that the above emotions, like disappointment, anger, caution and anxiety have the greatest correlation with social media fatigue. To some extent, they are "strains" in SSO model. As an "outcome", social media fatigue appears after these emotions accumulate to a certain extent. Subsequently, these users could clearly feel the relationship between role conflict and forced transformation of self-presentation strategies and these emotions. However, they sometimes did not consciously recognize their self-presentation strategies and their changing process on WeChat Moments. Their intuitive feeling was that their self-presentation gradually became "uncomfortable" and "not free". Similarly, they did not deliberately pay attention to the enlargement of the circle of contacts. In other words, it is difficult for these users to fully describe the relationship between "stress" and "strain" through self-report. However, the result of the interview has shown that they have actually been partially aware of the relationship between these factors. Thus, a problem has been raised that a further research may not rely too much on the self-report of the research object, otherwise it may lead to misjudgment of the correlation coefficient of the four factors.

Second, the article focuses on WeChat Moments and university students in mainland China. Although this has provided convenience for researchers, it also limits the representativeness of conclusions. Therefore, this leaves space for possible research in the future.

\section{REFERENCES}

[1] Dhir, A., Yossatorn, Y., Kaur, P., Chen, S. (2018) Online social media fatigue and psychological wellbeing-A study of compulsive use, fear of missing out, fatigue, anxiety and depression. International Journal of Information Management, 40: 141-152.

[2] Ravindran, T., Chua, A., Goh, D. (2013) Characteristics of Social Network Fatigue. In:10th International Conference on Information Technology: New Generations. Las vegas. pp.431-438.

[3] Bright, L., Kleiser, S., Grau, S. (2015) Too much Facebook? An exploratory examination of social media fatigue. Computers in Human Behavior, 44: 148-155.

[4] Hong, J., Duan, M. (2020) Social media fatigue and network social self under the generalization of one's WeChat Moments. Modern Communication (Journal of Communication University of China, 283: 76-85.

[5] Jiang, J. (2016) Generalization of WeChat Moment: Communication fatigue and emotional alienation. Modern Communication (Journal of Communication University of China), 241: 67-71.

[6] Wang, Y. (2019) Research on the unsustainable use of strong relationship social media from the perspective of anxiety -- Taking WeChat Moments as an example. Journalism and Mass Communication Monthly, 10: 81-91.

[7] Tencent Holdings Limited. (2021) 2021 interim report.

https://static.www.tencent.com/uploads/2021/08/31 /41f3fe664bf91be0400ad45eb0a233cd.pdf

[8] Xiao, L., Pan, T., Mou, J., Huang, L. (2020) Understanding determinants of social networking service fatigue: an interpretive structural modeling 
approach. Information Technology \& People.

[9] Goffman, E. (2008) The presentation of self in everyday life. Peking University Press, Beijing.

[10] Wang, J. (2017) Self-presentation of college students WeChat "Friends Circle". M. Southwest University. pp. 1-53

[11] Zhao, Q. (2019) Possible Impact Factors and Behavioral Consequences of Social Network Fatigue in Young People: A Relational Stress Perspective. Modern Communication (Journal of Communication University of China), 6: 59-75.

[12] Logan, K., Bright, L., Grau, S. (2018) UNFRIEND ME, PLEASE!": SOCIAL MEDIA FATIGUE AND THE THEORY OF RATIONAL CHOICE. Journal of Marketing Theory and Practice, 26: 357-367.

[13] Whelan, E., Islam, A., Brooks, S. (2020) Is boredom proneness related to social media overload and fatigue? A stress-strain-outcome approach. Internet Research, 30: 869-887.

[14] Zhang, Y., Liu, Y., LI, W., Peng, L., Yuan, C. (2020), A study of the influencing factors of mobile social media fatigue behavior based on the grounded theory. Information Discovery and Delivery, 48: 91-102.

[15] Xiao, L., Mou, J. (2019) Social media fatigue -Technological antecedents and the moderating roles of personality traits: The case of WeChat. Computers in Human Behavior, 101: 297-310.

[16] Zhang, Y, He, W \& Peng, L. 2020, 'How Perceived Pressure Affects Users' Social Media Fatigue Behavior: A Case on WeChat', Journal of Computer Information Systems, pp.1-11.

[17] Dhir, A., Kaur, P., Chen, S., Pallesen, S. (2019) Antecedents and consequences of social media fatigue. International Journal of Information Management, 48: 193-202.

[18] Zhang, Z., Chen, X., Deng, Y. (2021) From Immersed to Invaded, From Standby to Burnout:A Study on the Relationship between Work-Related Social Media Use During Non-Work Time and Job Burnout of Media Practitioners. Chinese Journal of Journalism \& Communication, 43: 160-176.

[19] Xue, J., Hong, J. (2020) Study on the Influencing Factors of Social Media Fatigue in Youth Groups from the Perspective of Role Stress: Taking WeChat Moments as an Example. Journalism and Mass Communication Monthly, 07: 21-32.

[20] Bossio, D., Holton, A. (2018) The identity dilemma: Identity drivers and social media fatigue among journalists. Popular Communication, 16:.248-262.

[21] Lee, C., Chou, S., Huang, Y. (2014) A Study on Personality Traits and Social Media Fatigue-Example of Facebook Users. Lecture Notes on Information Theory, 3: 249-253.

[22] Chen, Y. (2015) An Introduction to Communication Research Method. China Renmin University Press, Beijing.

[23] Reinard, J. (2008) Introduction to Communication Research. China Renmin University Press, Beijing.

[24] CNNIC. (2021) The $47^{\text {rd }}$ China statistical report on internet development. http://www.cnnic.net.cn/hlwfzyj/hlwxzbg/hlwtjbg/2 02102/P020210203334633480104

[25] Zhang, Q., Zhang, L. (2021) The Indeterminate Personality: The relationship between Digital Natives' Personality Traits and WeChat Profile Image. Chinese Journal of Journalism \& Communication, 43: 138-157.

[26] Cao, P., Yu, S. (2012) Proposal, research status and future development of digital aborigines. e-Education Research, 33: 21-27.

[27] Baker, S. (2021) How many qualitative interviews is enough? Expert voices and early career reflections on sampling and cases in qualitative research. National Centre for Research Methods Review Paper, pp. 1-42.

[28] Guo, Y. (2015) Friends zone of WeChat. M. Beijing University of Posts and Telecommunications, pp.1-89.

[29] Granovetter, M. (1973) The Strength of Weak Ties. American Journal of Sociology, 78: 1360-1380.

[30] Liu, Y. (2015) The behavioral analysis of 'impression management' on the WeChat Moments'. Journalism and Mass Communication Monthly, 03: 58-61,66.

[31] Fan, R. (2019) Analysis on the decline of user activity on WeChat Moments. North Media Research, 05: 66-69.

[32] John, L., Acquisti, A., Loewenstein, G. (2011) Strangers on a Plane: Context-dependent Willingness to Divulge Sensitive Information. Journal of Consumer Research, 37: 858-873. 